\title{
Original
}

\section{2 elevaciones de seno con técnica de ventana lateral y uso de biomaterial de relleno}

\author{
María Herrero*, Manuel Picón, Fernando Almeida, Leonardo Trujillo, \\ Jorge Núñez y Antonio Prieto
}

Sección de Cirugía Cervicofacial, Servicio de Cirugía Oral y Maxilofacial, Hospital Universitario Ramón y Cajal, Madrid, España

\section{INFORMACIÓN DEL ARTÍCULO}

Historia del artículo:

Recibido el 1 de febrero de 2011

Aceptado el 29 de mayo de 2011

Palabras clave:

Ventana lateral

Bio-Oss ${ }^{\circledR}$

Elevación seno

Atrofia maxilar

\section{Keywords:}

Lateral Window

Bio-Oss ${ }^{\circledR}$

Sinus floor augmentation

Maxillary atrophy

\section{R E S U M E N}

Introducción: Desde los estudios de Boyne y James y Tatum, la elevación de seno maxilar y colocación de injertos óseos se ha convertido en un procedimiento estándar que permite la colocación de implantes dentales en casos en los que la altura ósea posterior del maxilar es insuficiente. Aunque la utilización de hueso autólogo se considera la técnica gold-standard, en la actualidad se utilizan diversos biomateriales que obvian los inconvenientes de los injertos autólogos. Uno de los biomateriales más ampliamente utilizados es la matriz mineral ósea bovina desproteinizada en forma de gránulos (Bio-Oss ${ }^{\circledR}$ ).

Objetivos: Mostramos nuestra experiencia en implantología tras 382 elevaciones de seno.

Material y métodos: Presentamos el estudio de una serie de 382 casos de elevaciones de seno maxilar realizadas con técnica abierta y abordaje lateral utilizando Bio-Oss ${ }^{\circledR}$ como material de relleno y colocando implantes dentales simultáneamente o en una fase posterior, describiendo los protocolos utilizados y el seguimiento.

Resultados: Se realizaron un total de 382 casos (de los cuales 340 fueron en una sola fase y 42 en dos fases) durante el periodo comprendido entre octubre de 2002 y febrero de 2010 . Se colocaron un total de 726 implantes de los cuales fracasaron 27 (3,7\%).

Conclusiones: La elevación de seno maxilar para colocación de implantes dentales utilizando Bio-Oss ${ }^{\circledR}$ como material de relleno es una técnica fiable y con resultados predecibles a largo plazo como pone de manifiesto nuestro estudio.

(c) 2011 SECOM. Publicado por Elsevier España, S.L. Todos los derechos reservados.

\section{Sinus augmentation with a lateral window technique and use of a biomaterial filling}

\author{
A B S T R A C T
}

Background: Since the studies of Boyne and James research and then Tatum, maxillary sinus floor augmentation and grafting has become a standard procedure for dental implants in cases where the bone height in posterior maxilla it was insufficient. Although autogenous graft is considered the gold standard, there is currently a large amount of biomaterials which avoid the problems of autogenous bone grafts. One of the most used biomaterials is deproteinised bovine bone mineral matrix in granules (Bio- Oss ${ }^{\circledR}$ ).

\footnotetext{
* Autor para correspondencia

Correo electrónico: mariaherrero1982@gmail.com (M. Herrero).

1130-0558/\$ - see front matter @ 2011 SECOM. Publicado por Elsevier España, S.L. Todos los derechos reservados.

doi:10.1016/j.maxilo.2011.05.007
} 
Objective: The purpose of this study is to report our experience after 382 sinus augmentations with a lateral window approach and Bio-Oss ${ }^{\circledR}$ graft.

Material and Methods: The clinical records from 382 patients having posterior maxillary atrophy treated with lateral approach sinus floor augmentation, Bio-Oss ${ }^{\circledR}$ material graft and simultaneous or delayed dental implants. The technique used and short- term postoperative results were reviewed.

Results: A total of 382 patients were treated (of which 340 were done in a single phase and 42 in two phases) during the period from October 2004 to January 2010. A total of 726 implants were made, of which 27 (3.7\%) failed.

Conclusions: Our study shows that maxillary sinus augmentation with Bio-Oss ${ }^{\circledR}$ and lateral window approach seems to be a reliable technique with predictable long-term results.

(c) 2011 SECOM. Published by Elsevier España, S.L. All rights reserved.

\section{Introducción}

La atrofia avanzada del maxilar y la neumatización consecuente del seno maxilar comportan un reto para la rehabilitación implantosoportada. La técnica de elevación de seno maxilar se ha convertido en un procedimiento estándar que permite la colocación de implantes dentales en casos en los que la altura ósea del maxilar posterior es insuficiente.

En la actualidad se prioriza la búsqueda en dicha técnica de métodos menos invasivos, más simples, que conlleven menos complicaciones asociadas y que alcancen objetivos implantológicos y protésicos posteriores en menor tiempo.

En 1970 Tatum $^{1}$ describe la primera técnica de elevación subantral por abordaje externo con ventana lateral, abriendo una ventana lateral intentando no romper la membrana de Schneider y creando un espacio entre el suelo sinusal y la misma rellenándolo con hueso autólogo o biomateriales. En 1980 esta técnica fue modificada por Boyne y James² ${ }^{2}$, siendo ampliamente utilizada después ${ }^{3,4}$.

Misch en 1984 mejoró la técnica de relleno subantral con hueso autólogo descrita anteriormente por Boyne y James e introduce una clasificación según variaciones anatómicas antrales ${ }^{5}$.

Summers en 1994 describe la técnica con abordaje transalveolar o aproximación a la membrana de Schneider mediante osteotomos desde la cresta dejando 1-2 mm de hueso hasta suelo sinusal. Esta distancia de hueso se eleva mediante presión empujando la membrana hacia arriba sin perforarla y creando espacio para alojar biomateriales e implante ${ }^{6}$.

Experiencias como la de Fugazzoto y Vlassis en 1998 con un seguimiento a 6 años en 222 elevaciones de seno con 510 implantes ${ }^{7}$ y Aghaloo y Moy ${ }^{9}$, o Jurisic et $\mathrm{al}^{8}$ con un seguimiento a 3 años en 61 elevaciones de seno y 80 implantes valorando ambas técnicas de abordaje y obteniendo tasas de éxito similares, sostienen la tendencia actual en la literatura sobre la independencia del éxito implantológico en relación a la técnica de abordaje utilizada.

El hueso autólogo se ha considerado el material ideal para relleno tras la elevación de seno maxilar al ser un material osteoproductivo eficaz que facilita la regeneración ósea. Sin embargo, los autoinjertos presentan varios problemas o desventajas como su escasez, su reabsorción y la morbilidad del lugar donante por lo que cada vez se utilizan más diversos biomateriales con propiedades osteoconductivas, siendo la matriz mineral ósea bovina desproteinizada (Bio-Oss ${ }^{\circledR}$ ) uno de los más utilizados ${ }^{10-12}$.

La matriz ósea bovina (Bio-Oss ${ }^{\circledR}$ ) ha demostrado favorecer la osteointegración implantológica gracias a su lenta reabsorción. Y es ampliamente utilizada como material de relleno sustitutivo óseo gracias a sus resultados favorables ${ }^{13-17}$.

Las membranas de barrera (de colágeno en nuestro caso) se usan fundamentalmente para reparar perforaciones en la membrana sinusal y para proteger el acceso al seno, colocadas sobre el orificio de la antrostomía. Su utilización en conjunción con el biomaterial de relleno favorece un mejor aislamiento del mismo además de la disminución de la osteoclastogénesis ${ }^{18,19}$.

\section{Material y métodos}

Presentamos un estudio retrospectivo de una serie de 382 elevaciones de seno realizadas en 342 pacientes durante un periodo de 7 años y 5 meses (octubre 2002- febrero 2010). El criterio de inclusión fue altura ósea maxilar posterior igual o menor de $6 \mathrm{~mm}$. La edad media fue de 62 años con un rango de 18 a 83 años. De los 342 pacientes 221 eran mujeres (64,62\%) y $121(35,38 \%)$ hombres. Algunos presentaban factores de riesgo (considerados en nuestra serie): 32 eran fumadores de más de 20 UPA, 8 estaban en tratamiento anticoagulante con Sintrom, 25 eran diabéticos, 20 mujeres estaban en tratamiento con bifosfonatos orales (los cuales fueron suspendidos durante 4 meses previos a la realización de la técnica, según señalan las directrices de la SECOM) ${ }^{20}$ y 1 fue rechazado por encontrarse en lista de espera para ser trasplantado de pulmón.

A todos los pacientes se les realizó un estudio preoperatorio que incluye una exploración intraoral inicial, un estudio radiográfico con ortopantomografía (OPG) y Denta- Scan, realización de modelos oclusales y una encuesta de salud. Todos ellos firman consentimiento informado del proceso quirúrgico (fig. 1).

De los 342 pacientes en 302 (79,05\%) se realizó elevación de seno unilateral y en $40(10,47 \%)$ se realizó de forma bilateral (80 elevaciones de seno). En 342 (89,53\%) de las elevaciones realizadas los implantes se colocaron de forma simultánea a la elevación (una fase) y en 40 (10,47\%) los implantes se colocaron de forma diferida 4 meses después de la elevación. Los criterios para realizar la técnica en una o dos fases fueron la altura del reborde alveolar remanente y la estabilidad primaria del 




Figura 1 - Denta-Scan con modelos implantológicos preliminares.

implante. Si la altura ósea era mayor de $4 \mathrm{~mm}$ y la estabilidad primaria del implante adecuada según el criterio clínico del cirujano se realizaba en una fase. Si la altura ósea era menor de $4 \mathrm{~mm}$ y/o la estabilidad primaria del implante inadecuada, se realizaba en dos fases. Cuando esta se realizaba se colocaban los implantes osteointegrados mediante técnica transmucosa.

Se colocaron implantes roscados, cilíndricos, con recubrimiento RBM (procedimiento de alteración de la superficie del implante que no utiliza el grabado al ácido, por lo que la superficie de los implantes RBM se encuentra, por definición, libre de residuos de grabado al ácido) y hexágono externo.

Para evitar la invasión del tejido conectivo desde el mucoperiostio colocamos dos membranas de colágeno; una interna y otra externa (fig. 2).

Como medida física todos utilizaron hielo local. Como fármacos, AINES o paracetamol a demanda y antibioterapia profiláctica durante 4 días con augmentine $500 \mathrm{mg} / 8 \mathrm{~h}$. Aquellos que son alérgicos a penicilinas utilizaron clindamicina (dalacín) $250 \mathrm{mg} / 6 \mathrm{~h}$.

Cuando los pacientes eran portadores de prótesis removibles se les aconsejó no utilizarlas o utilizarlas durante en menor tiempo posible durante las primeras semanas y

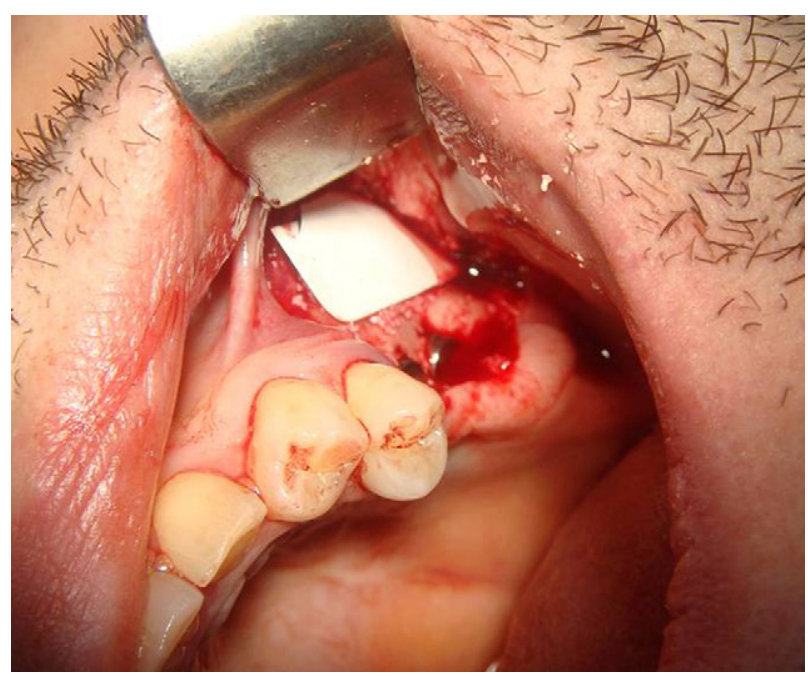

Figura 2 - Técnica de elevación de seno con membrana de colágeno. siempre bajo la estricta vigilancia de su odontólogo para evitar cargas indebidas sobre los implantes o las zonas injertadas.

La retirada de puntos se realizó a los 10 días postoperatorios.

Se realizó un seguimiento al mes con revisión clínica del paciente, a los dos meses y medio una nueva revisión clínica y realización de OPG de control, y a los cuatro meses, una vez comprobada clínicamente la integración de los implantes, los pacientes fueron remitidos al prostodoncista para la carga. En un $64 \%$ de los pacientes tuvimos que abrir los implantes y colocar tapones de cicatrización. En el resto de los casos la exposición espontánea de los implantes lo hizo innecesario.

Los pacientes fueron remitidos para la toma de impresiones a los 4 meses por lo que la carga efectiva de los implantes se realizó a los 5 meses de promedio.

Después de la carga los pacientes fueron revisados por el prostodoncista a los 1, 3, 6 y 12 meses y anualmente después.

\section{Resultados}

Realizamos un total de 382 elevaciones de seno en 342 pacientes, de los cuales 221 eran mujeres y 121 eran hombres. Con una media de 62 años de edad, en un rango de 18 a 83 años de edad.

Se colocaron 726 implantes en total, de los cuales 632 lo fueron en una sola fase y 94 de forma diferida. Las medidas de los implantes y su posición se especifican en la tabla 2.

Sobre los 726 implantes se colocaron 774 coronas.

El periodo de seguimiento medio fue de 27 meses con un máximo de 88 meses y un mínimo de 6 meses.

El prostodoncista realizó un seguimiento al mes, a los 2 meses y medio y a los 3 meses y medio. En total se ha realizado un seguimiento medio de 27 meses con un máximo de 88 meses y un mínimo de 3 meses en los pacientes incluidos en la serie en el mes de febrero.

Obtuvimos un 3,7\% de fracasos, con 27 implantes fracasados. Uno en primer premolar, 4 en el segundo, 10 en el primer molar y 12 en el segundo molar. Antes de la carga fracasaron 15 implantes y después de la carga 12.

Entre los incidentes encontrados: en 5 pacientes fue abortado el proceso: 3 casos por hemorragia masiva al realizar la ostectomía (2 de los cuales rechazaron un segundo intento y a 1 se le realizó 6 meses más tarde sin ninguna complicación) y en 2 casos por fenestración masiva de la membrana de Schneider que no se pudo reparar (el procedimiento se les realizó 6 meses más tarde sin complicaciones asociadas).

En 63 pacientes $(18,42 \%)$ hubo perforaciones menores de la mucosa, que se resolvieron en su totalidad con el uso de la membrana de colágeno.

En 53 pacientes la primera consulta se realiza a petición propia antes de los 10 días con 14 incidencias por sangrado débil por la mucosa oral, 8 con sangrado por fosa nasal, 44 hematomas o inflamación excesiva, 17 casos de eliminación de restos de material de relleno (6 en fosa nasal, 11 en cavidad oral). En 1 caso, en el que el paciente era alérgico a penicilinas y estaba en tratamiento con dalacín, consultó por rinorrea unilateral, resolviéndose el cuadro con un tratamiento con ciprofloxacino oral durante 6 días. En ninguno de estos casos hubo que retirar el material de relleno ni los implantes. 


\section{Discusión}

Actualmente son muchas las técnicas descritas para la rehabilitación de la atrofia de maxilar posterior, basadas en distintos procedimientos de remodelación de la propia cresta, o utilizando diversos tipos y técnicas de injerto óseo, favoreciendo con ello, la colocación inmediata o diferida de los implantes. Una de estas técnicas es la técnica de elevación sinusal.

A pesar de que la incisión crestal levemente palatinizada está recogida como la más adecuada pues discurre entre los territorios vasculares bucal y palatinos respetándolos, en nuestra serie de 382 pacientes utilizamos la técnica de abordaje de ventana lateral en todos los casos demonstrándo ser ésta una técnica fiable y de fácil aplicación en manos de cirujanos experimentados. Otras series como las de Fugazzoto y Vlassic o las de Jurisic et al lo corroboran ${ }^{7,8}$. Las descargas deben de alejarse de la zona de la osteotomía aproximadamente en $1 \mathrm{~cm}$.

En el manejo y colocación del injerto, diversos autores recomiendan, sobre todo en xenoinjertos, el uso de mezcla de partículas de pequeño y gran tamaño para producir un espacio interparticulado adecuado y mantener así una alta capacidad de osteoconducción y menor reabsorción ${ }^{21}$.En nuestra serie, donde hemos empleado matriz bovina ósea desmineralizada, la mayoría eran de pequeño tamaño y en ningún caso esto ha impedido el crecimiento vascular a través del injerto.

En relación con la técnica, una de las dificultades (la más frecuente en las distintas series recogidas en la literatura) es la disección de la membrana sinusal de Schneider, cuya perforación puede presentar ciertas complicaciones, no siendo estas determinantes en la supervivencia o el fracaso de los implantes posteriores si no son masivas ${ }^{22,23}$. En nuestra serie se produjo en $63(18,42 \%)$ de los pacientes, no presentando ninguna complicación posterior con la aposición de la membrana de colágeno intraoperatoria en el momento de la perforación. Sin embargo, la perforación masiva de la membrana nos ocurrió en 2 de 5 pacientes en los que la técnica tuvo que ser suspendida. Lo que supondría un $40 \%$ en nuestra serie de causas por las que el proceso se abortó.

En el último consenso nacional sobre injerto óseo maxilar se estableció el xenoinjerto como material de elección, con el propósito de la osteointegración de los implantes, siendo efectivos incluso en senos gravemente atróficos. La necesidad de añadir hueso autólogo a los mismos es controvertida ${ }^{21}$.

En nuestra serie obtuvimos un éxito implantológico de un $96,4 \%$ con material xenogénico implantado sin añadir hueso autólogo.

Las membranas de barrera se utilizan para proteger al muco- periostio vestibular, y previenen la inmigración de células no osteogénicas ${ }^{18,19,21}$.

En nuestros 382 pacientes se utilizaron con buenos resultados y con ellas se resolvieron las perforaciones de la membrana de Schneider.

No existe suficiente evidencia que certifique que el plasma rico en plaquetas o en factores de crecimiento usado de forma simultánea con el material xenogénico estimule la regeneración ósea ${ }^{17,21}$

La estabilidad inicial del implante es el factor clave para la osteointegración y debe ser el principal criterio para indicar implantes simultáneos o diferidos en el seno maxilar. La osteointegración del implante está directamente relacionada con la calidad del hueso donde va a insertarse y la superficie del implante. En nuestros pacientes el establecimiento de implantes en dos fases o diferidos viene condicionada por una altura inicial de reborde alveolar menor de $4 \mathrm{~mm}$ (medida por Denta-Scan inicial) o ausencia de estabilidad primaria. Otras series establecen una altura mínima de reborde alveolar en torno a 4-5 $\mathrm{mm}$ para la implantación en una sola fase ${ }^{21,24}$.

La importancia de nuestra serie es el volumen considerable unicéntrico de pacientes que han sido sometidos a una elevación de seno e implantología con un seguimiento de 7 años y 5 meses con una alta tasa de éxito implantológico. Además de una técnica mínimamente invasiva para el paciente así como una rápida ejecución, con 30 minutos de media en su duración.

$\mathrm{Al}$ igual que sucede en muchos otros estudios una de las limitaciones que encontramos en nuestra serie de casos es la falta de randomización y control de asociación entre variables cuantitativas y cualitativas con la técnica y el uso de biomaterial.

A pesar de que la técnica de elevación de seno con xenoinjertos en maxilares posteriores atróficos se presenta como una solución válida para la implantología, son necesarios estudios prospectivos más uniforme en sus parámetros de evaluación para facilitar la experiencia particular de los profesionales en dicha técnica, además de estudios comparativos entre materiales sustitutivos óseos y nuevas opciones en desarrollo como proteínas morfogenéticas y cultivos óseos celulares.

\section{Conclusiones}

La técnica de elevación de seno para resolver casos de falta de altura en la colocación de implantes en maxilares posteriores atróficos presenta una alta previsibilidad de éxito reforzada por la medicina basada en la evidencia. Al mismo tiempo el mineral óseo bovino (Bio-Oss ${ }^{\circledR}$ ) ha demostrado ser un sustituto óseo que favorece la osteointegración implantológica gracias a su lenta reabsorción y fácil manejo.

La combinación de Bio-Oss ${ }^{\circledR}$ con membrana de colágeno y técnica de abordaje lateral presenta resultados favorables en nuestra serie.

Por otra parte la escasa morbilidad y el bajo índice de complicaciones derivadas de la técnica, hacen de ella una técnica recomendable cuando esté indicada.

\section{Conflicto de intereses}

Los autores declaran no tener ningún conflicto de intereses.

\section{B I B L I O GRAF Í A}

1. Emmerich D, Att W, Stappert C. Sinus floor elevation using osteotomes: a systematic review and meta-analysis. J Periodontol. 2005;76:1237-51.

2. Boyne PJ, James RA. Grafting of the maxillary sinus floor with autogenous marrow and bone. J Oral Surg. 1980;38:613-6. 
4. Lorenzetti M, Mozzati M, Campanino PP, Valente G. Bone augmentation of the inferior floor of the maxillary sinus with autogenous bone or composite bone grafts a histologicistomorphometric preliminary report. Int J Oral Maxillofac Implants. 1998;13:69-76.

3. Raghoebar GM, Vissink A, Reintsema H, Batenburg RH. Bone grafting of the floor of the maxillary sinus for the placement of endosseous implants. Br J Oral Maxillofac Surg. 1997;35:119-25.

5. Misch CE. Bone Classification, training keys to implant success. Dent Today. 1989;8:39-44.

6. Summers RB. A New Concept in maxillary implant surgery: the osteotome technique. Compendium. 1994;15: 154-6.

7. Fugazzotto PA, Vlassis J. Long-term success of sinus augmentation using various surgical approaches and grafting materials. Int J Oral Maxillofac Implants. 1998;13:52-8.

8. Jurisic M, Markovic A, Radulovic M, Brkovic BM, Sdndor GK. Maxillary sinus floor augmentation: comparing osteotome with lateral window immediate and delayed implant placements. An interim report. Oral Surg Oral Med, Oral Pathol, Oral Radiol Endod. 2008;106:820-7.

9. Aghaloo TL, Moy PK. Which hard tissue augmentation techniques are the most successful in furnishing bony support for implant placement? Int J Oral Maxillofac Implants. 2007;22 Suppl:49-70.

10. Kent JN, Block MS. Decisión making for sinus graft surgery: Techniques based in clinical and histologic success. American Academy of osseointregation fifteenth annual meeting. New Orleans. March $10^{\text {th }} 2000$.

11. Hall HD, Mckenna SJ. Maxillary sinus augmentation using different grafting materials and dental implants in monkeys. Part I. Evaluation of porous hydroxyapatite. Clin Oral Impl Res. 1997;8:847-96.

12. Hurzeler M, Quiñones C, Kirsch A. y cols. Maxillary sinus augmentation using different grafting materials and dental implants in monkeys. Part I. Evaluation of autogenous bone combined with hydroxylapatite. Clin Oral Impl Res. 1997;8:401-11.

13. Valentini $P$, Abensur DJ. Maxillary sinus grafting with anorganic bovine bone:a clinical report of long-term results. Int J Oral Maxillofac Implants. 2003 Jul-Aug;18:556-60.

14. Szabó G. A prospective multicenter randomized clinical trial of autogenous bone versus beta- tricalcium phosphate graft alone for bilateral sinus elevation: histologic and histomorphometric evaluation. Int J Oral Maxillofac Implants. 2005 May-Jun;20:371-81.

15. Froum SJ, Tarnow DP, Wallace SS, Rohrer MD. Chosc. Sinus floor elevation using anorganic bovine bone matrix (OsteograN) with and without autogenous bone: a clinical, histologic, radiographic, and histomorphometric analysis. Int J Periodontics Restorative Dent. 1998 Dec;18:528-43.

16. Nkenke F, Stelze F. Clinical outcomes of sinus floor augmentation for implant placement using autogenous bone or bone substitutes: a systematic review. Clin Oral Implants Res. 2009 Sep;20 suppl 4:124-33.

17. Broways H, Brouvry P, De Bruyn H. A literature Review on Biomaterials in Sinus Augmentation Procedures. Clin Impl Dent. 2007;9:166-77.

18. Sculean A, Nikoliadis D, Schwarz F. Regeneration of periodontal tissues: combinations of barrier membranes and grafting materials- biological foundations and preclinical evidence: a systematic review. J Clin Periodontal. 2008 sep;35:106-16.

19. Agis H, Magdalenko M, Stögerer K, Watzek G, Gruber R. Collagen barrier membranes decrease osteoclastogenesis in murine bone marrow cultures. Clin Oral Implants Res. 2010;21:656-61.

20. Junquera LM, Martin- Granizo R. Diagnóstico, Prevención y tratamiento de la osteonecrosis de los maxilares por bifosfonatos. Recomendaciones de la SECOM. Rev Esp Cirug Oral y Maxilofacial. 2008;30:145-56.

21. Villarreal PM, Fernández-Bustillo A, Acero J, Arruti JA, Baladrón J, Bilbao A, et al. I Conferencia Nacional de Consenso sobre el Injerto Óseo del Seno Maxilar. Rev Esp Cirug Oral y Maxilofac. 2010;32:41-94.

22. Wallace SS, Mazor Z, Frorum SJ, Cho SC, Tarnow DP. Schneiderian membrane perforation rate during sinus elevation using piezosurgery: clinical results of 100 consecutive cases. Int J Periodontics Restorative Dent. 2007;27:413-9.

23. Anavi Y, Allon DM, Avishai G, Calderon S. Complication of maxillary sinus augmentations in a selective series of patients. Oral Surg Oral Med Oral Pathol Oral Radiol Endod. 2008;106:34-8.

24. Gui-Youn CL, Naval-Gías L, Mancha de la Plata M, SastrePérez J, Capote Moreno AL, Muñoz- Guerra MF. Elevación de seno maxilar. Análisis clínico de nuestra experiencia en más de 100 casos. Rev Esp Cir Oral Maxilofac. 2009;31:223-30. 